\title{
Deep Imaging of SNRs at Low Frequencies using the GMRT
}

\author{
Sanjay Bhatnagar \\ National Centre for Radio Astrophysics, TIFR, Pune, India
}

\begin{abstract}
Radio observations of large Supernova Remnants (SNRs) in the Galaxy are plagued with the problems of confusion and limitations of synthesis telescopes in imaging the emission at large angular scales. Since most SNRs are brighter at low radio frequencies, and the contamination due to thermal emission is reduced, deep and high resolution imaging of Galactic SNRs at meterwave lengths is most useful in their detection and classification. Relatively high resolution and sensitivity over a large range of angular scales provided by the GMRT at meterwave lengths makes it an ideal instrument for detailed multi-frequency imaging of Galactic SNRs.
\end{abstract}

We report 327-MHz observations of three large $\left(>15^{\prime}\right)$ candidate supernova remnants (SNRs) at high Galactic latitudes using the Giant Meterwave Radio Telescope (GMRT). We detect extended 327-MHz emission in all three fields, which were proposed as candidate SNRs by Duncan et al. (1997, MNRAS, 287,722 ) in the Parkes $2.4-\mathrm{GHz}$ survey. G356.2+4.5 has a well-resolved shell of emission and contains a $32 \mathrm{mJy}$ pulsar situated at a distance of $1.43 \mathrm{kpc}$ as derived from the pulsar dispersion measure. The field of G358.0+3.8 is highly confused and although we confirm the presence of a partial ring, it is a lower signal-to-noise ratio detection. G004.8+6.2 (formerly G4.5+6.2) is located $\approx$ $40^{\prime}$ away from Kepler's SNR and we detect a $17^{\prime} \times 18^{\prime}$ SNR, possibly of shell morphology. All these fields show clear, well-resolved shells in the NRAO VLA Sky Survey (NVSS) maps, which are also presented. Table gives the measured and derived parameters of the three SNRs presented here. The spectral indices were measured from the flux densities at 4.85 and $2.4 \mathrm{GHz}$ from the PMN and Parkes surveys respectively, and the $327-\mathrm{MHz}$ GMRT observations. We did not use the flux densities from NVSS because of missing large scale emission in these images. Spectral index for G358.0+3.8 was not determined due to possible missing emission in the $327-\mathrm{MHz}$ map as well thermal component in the 4.85 and 2.4-GHz maps.

\begin{tabular}{lcccccc}
\hline Name & $\begin{array}{c}\text { RA } \\
(\mathrm{J} 2000)\end{array}$ & $\begin{array}{c}\text { Dec } \\
(\mathrm{J} 2000)\end{array}$ & $\begin{array}{c}\text { Size } \\
\left({ }^{\prime}\right)\end{array}$ & $\begin{array}{c}S_{327} \\
(\mathrm{Jy})\end{array}$ & $\begin{array}{c}\text { RMS } \\
(\mathrm{mJy} /) \\
\text { beam })\end{array}$ & $\begin{array}{c}\text { Sp. Index } \\
\left(S \propto \nu^{\alpha}\right)\end{array}$ \\
\hline G356.2+4.5 & $17^{\mathrm{h}} 18^{\mathrm{m}} 58^{\mathrm{s}}$ & $-29^{\circ} 40^{\prime}$ & 25 & $8.1 \pm 1.7$ & 10 & $-0.66 \pm 0.17$ \\
$\mathrm{G} 358.0+3.8$ & $17^{\mathrm{h}} 26^{\mathrm{m}} 04^{\mathrm{s}}$ & $-28^{\circ} 36^{\prime}$ & 38 & $2.5 \pm 1.3$ & 15 & - \\
$\mathrm{G} 4.8+6.2$ & $17^{\mathrm{h}} 33^{\mathrm{m}} 25^{\mathrm{s}}$ & $-21^{\circ} 34^{\prime}$ & 18 & $5.5 \pm 1.2$ & 23 & $-0.57 \pm 0.13$ \\
\hline
\end{tabular}

Table 1. Parameters of the SNRs at $327 \mathrm{MHz}$. 

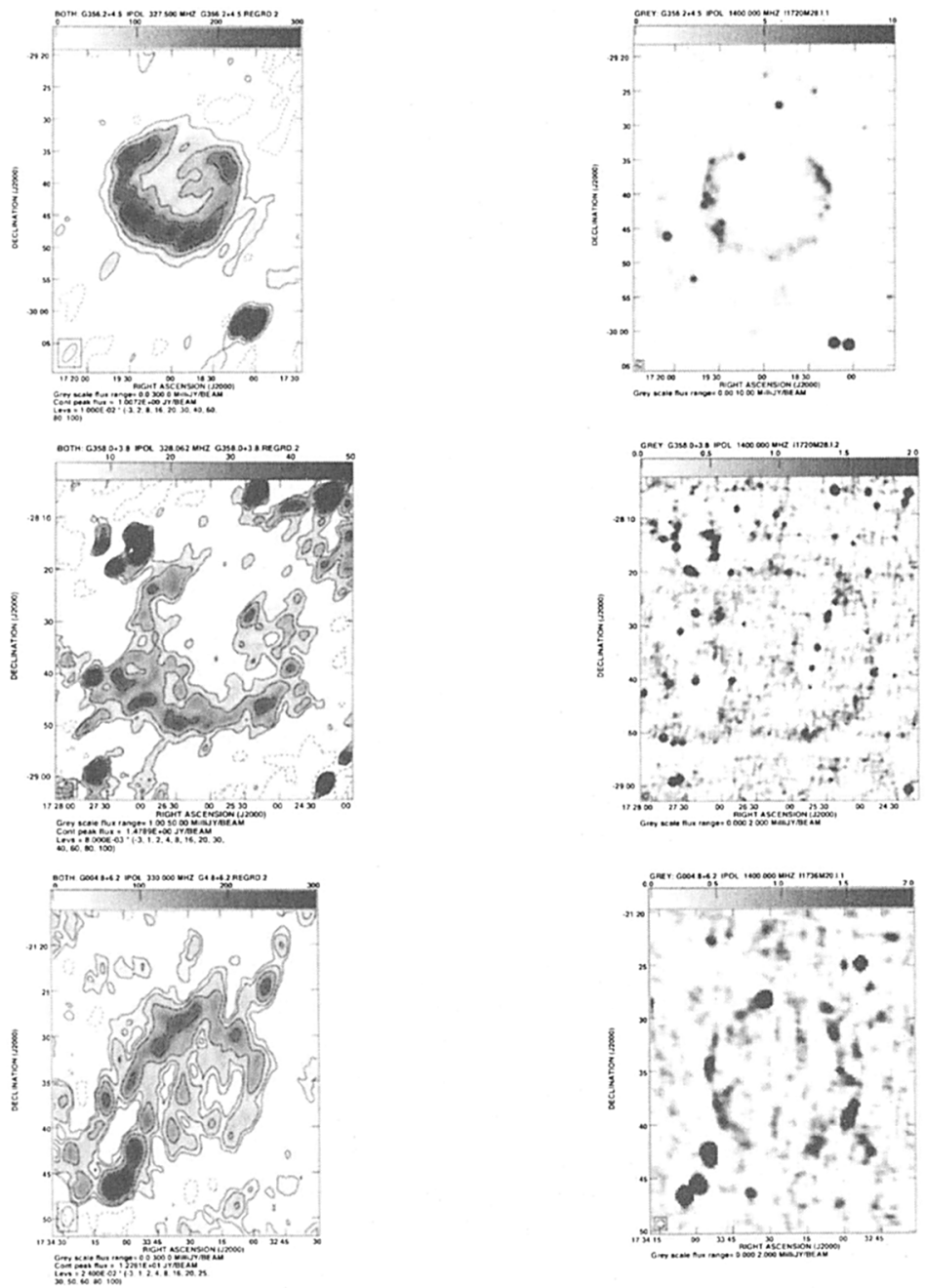

Figure 1. Left hand panels show the GMRT $327-\mathrm{MHz}$ images of G356.2+4.5, G358.0+3.8 and G004.8+6.2 respectively. The right hand panels show the NVSS $1400-\mathrm{MHz}$ images of these SNRs. 